\title{
Cellular-Resolution Imaging of Microstructures in Rat Bone using Laboratory Propagation-Based Phase-Contrast X-ray Tomography
}

\author{
$\underline{\text { Ilian Häggmark }}{ }^{1, *}$, Jenny Romell ${ }^{1}$, Susanne Lewin ${ }^{2}$, Caroline Öhman ${ }^{2}$, and Hans M. Hertz ${ }^{1}$ \\ 1. Dept. of Applied Physics, KTH Royal Institute of Technology, Stockholm, Sweden. \\ 2. Dept. of Engineering Sciences, Uppsala University, Uppsala, Sweden. \\ * Corresponding author, ilian.haggmark@biox.kth.se
}

Imaging of the bone microstructure is essential for analyzing the processes that govern bone remodeling, i.e. the resorption and formation of bone tissue. This is for instance relevant for understanding osteoporosis and fracture healing [1]. Propagation-based phase-contrast X-ray imaging is suited for imaging biological microstructures since it is sensitive to high spatial frequencies and low-absorbing materials. Britz et al have previously shown the microstructure in rat bones using synchrotron radiation micro-computed tomography [2]. The limited access to synchrotron facilities makes comprehensive studies and future diagnostic use difficult. Here we show that similar image quality can be obtained using a compact laboratory system.

The porosity in cortical bone microstructure includes the vascular canal system and the osteocyte lacunar system. Vascularization in bone controls oxygen and nutrient delivery, vital for many physiological processes, such as bone remodeling and healing. Osteocytes are bone cells, also highly involved in these processes. In the small cavities called lacunae, osteocytes are spread through the whole bone, connected in a network by a canal system (canaliculi). Osteocytes sense mechanical loading, and initiate adaptive remodeling of the bone structure [2]. Assessment of alterations in cortical bone microstructure is used to study diseases, for example bone degenerative diseases such as osteoporosis, or metabolic disorders such as diabetes. Consequently, microstructure imaging and quantification are of high importance to increase the knowledge of bone development and remodeling.

A rat femur, which is part of a study on biomaterials for bone regeneration [3], was imaged using a liquidmetal-jet microfocus source (MetalJet D2, Excillum AB) [4]. The acceleration voltage was $70 \mathrm{kV}$ and the spectrum was filtered with $0.5 \mathrm{~mm}$ aluminum, yielding a mean energy of $29 \mathrm{keV} .2000$ projections with angular step $0.1^{\circ}\left(180^{\circ}+\right.$ cone angle is sufficient) were acquired using a Photonic Science FDI-VHR detector with a $4008 \times 2671$ pixel CCD and a $15 \mu \mathrm{m}$ thick Gadox $\left(\mathrm{Gd}_{2} \mathrm{O}_{2} \mathrm{~S}: \mathrm{Tb}\right)$ scintillator. The voxel size was $1.9 \mu \mathrm{m}$, effective propagation distance $0.17 \mathrm{~m}$ and field of view $\sim 7.5 \times 7.5 \times 5.0 \mathrm{~mm}^{3}$. The exposure time for each projection was 2 minutes. The images were flat-field corrected, phase retrieved [5] and finally reconstructed in Octopus Reconstruction 8.9. Further visualization was carried out in Amira 6.3.

Figure 1(a) shows an axial slice of the rat femur. Vascular canals, approximately $10-15 \mu \mathrm{m}$ in diameter, are clearly visible in the cortical bone. Figure 1(b) shows a magnified part of the cortical bone. Here the concentric configuration of lacunae can be observed (A). Mergers and splits of vascular canals are also visible (B). This can also be seen in a longitudinal slice (see Fig. 1(c)). The full branching of the vascular canals can be studied by segmentation (see Fig. 2), which allows for a quantitative analysis.

We have shown that bone microstructures can be imaged with cellular resolution and reasonable field of view using laboratory X-ray phase-contrast setups. This can enable more comprehensive studies of bone microstructure, particularly in relation to bone disease and fracture healing [6]. 
References:

[1] I S Maggiano, et al, J. Anat. 228 (2016) p. 719.

[2] H M Britz et al, Bone 51 (2012) p. 913.

[3] G Hulsart-Billström et al, J. Tissue Eng Regen Med 9 (2012) p. 799.

[4] O Hemberg et al, Appl. Phys. Lett. 83 (2003) p. 1483.

[5] D Paganin et al, J. Microsc. 207 (2002) p. 33.

[6] The authors acknowledge funding from the Swedish Research Council and the Wallenberg Foundation.

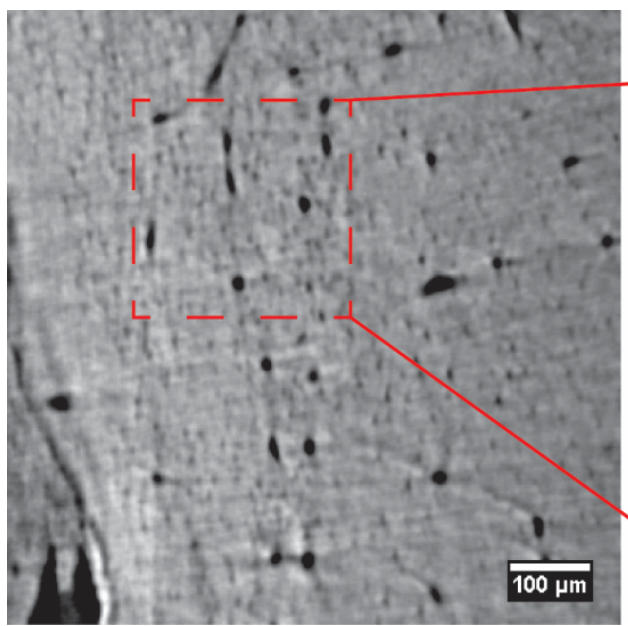

(a)

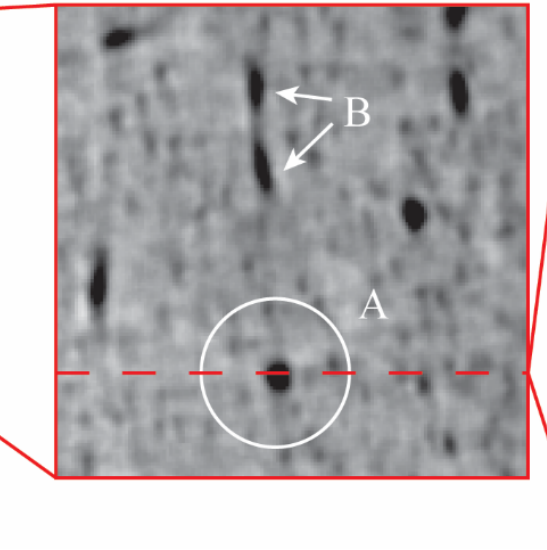

(b)

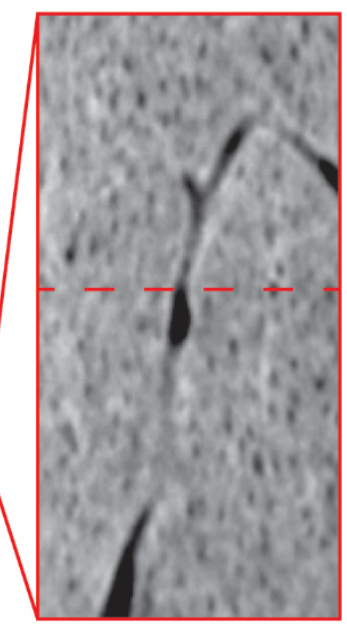

(c)

Figure. 1. (a) Axial slice of cortical bone in a rat femur. (b) Part of cortical bone where structures such as vascular canals and lacunae are visible. (A) Concentric patter of lacunae with vascular canal in center, (B) branching of vascular canals. (c) Longitudinal slice where branching of canals is visible.

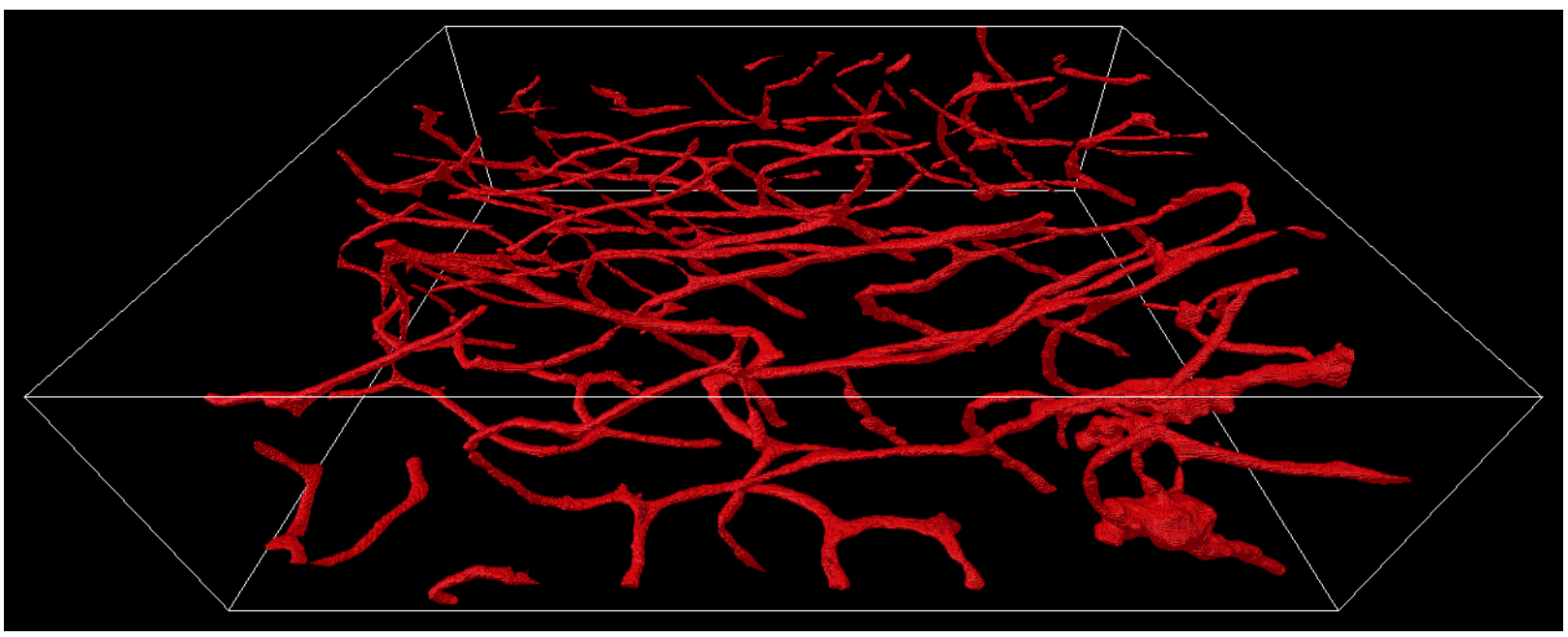

Figure. 2. 3D rendering of the vascular canals. The white bounding box is $380 \times 950 \times 1320 \mu \mathrm{m}^{3}$. The axial direction of the bone is horizontal in the image. 